\title{
Binary Relations-based Rough Sets - an Automated Approach
}

\author{
Adam Grabowski \\ Institute of Informatics \\ University of Białystok \\ Ciołkowskiego 1M, 15-245 Białystok \\ Poland
}

Summary. Rough sets, developed by Zdzisław Pawlak [12, are an important tool to describe the state of incomplete or partially unknown information. In this article, which is essentially the continuation of [8], we try to give the characterization of approximation operators in terms of ordinary properties of underlying relations (some of them, as serial and mediate relations, were not available in the Mizar Mathematical Library [11]). Here we drop the classical equivalence- and tolerance-based models of rough sets trying to formalize some parts of [18.

The main aim of this Mizar article is to provide a formal counterpart for the rest of the paper of William Zhu [18. In order to do this, we recall also Theorem 3 from Y.Y. Yao's paper [17. The first part of our formalization (covering first seven pages) is contained in 8]. Now we start from page 5003, sec. 3.4. 18. We formalized almost all numbered items (definitions, propositions, theorems, and corollaries), with the exception of Proposition 7, where we stated our theorem only in terms of singletons. We provided more thorough discussion of the property positive alliance and its connection with seriality and reflexivity (and also transitivity). Examples were not covered as a rule as we tried to construct a more general mechanism of finding appropriate models for approximation spaces in Mizar providing more automatization than it is now [10.

Of course, we can see some more general applications of some registrations of clusters, essentially not dealing with the notion of an approximation: the notions of an alliance binary relation were not defined in the Mizar Mathematical Library before, and we should think about other properties which are also absent but needed in the context of rough approximations [9], 5]. Via theory merging, using mechanisms described in [6] and [7, such elementary constructions can be extended to other frameworks. 


\section{MSC: 03E70 03E99 03B35}

Keywords: rough set; lower approximation; upper approximation; binary relation

MML identifier: ROUGHS_3, version: 8.1.04 5.36.1267

\section{Preliminaries}

From now on $X, a, b, c, x, y, z, t$ denote sets and $R$ denotes a binary relation.

Let $X$ be a non empty set. Let us note that $2^{X}$ is closed under directed unions.

The scheme FinSubIndA1 deals with a non empty, finite set $\mathcal{X}$ and a unary predicate $\mathcal{P}$ and states that

(Sch. 1) For every subset $B$ of $\mathcal{X}, \mathcal{P}[B]$

provided

- $\mathcal{P}\left[\emptyset_{\mathcal{X}}\right]$ and

- for every subset $B^{\prime}$ of $\mathcal{X}$ and for every element $b$ of $\mathcal{X}$ such that $\mathcal{P}\left[B^{\prime}\right]$ and $b \notin B^{\prime}$ holds $\mathcal{P}\left[B^{\prime} \cup\{b\}\right]$.

The scheme FinSubIndA2 deals with a non empty, finite set $\mathcal{X}$ and a unary predicate $\mathcal{P}$ and states that

(Sch. 2) For every non empty subset $B$ of $\mathcal{X}, \mathcal{P}[B]$

provided

- for every element $x$ of $\mathcal{X}, \mathcal{P}[\{x\}]$ and

- for every non empty subsets $B_{1}, B_{2}$ of $\mathcal{X}$ such that $\mathcal{P}\left[B_{1}\right]$ and $\mathcal{P}\left[B_{2}\right]$ holds $\mathcal{P}\left[B_{1} \cup B_{2}\right]$.

Let us consider a function $f$ and sets $a, y$. Now we state the propositions:

(1) Suppose $\operatorname{dom} f$ is subset-closed and closed under directed unions and $f$ is preserving directed unions. Then if $a \in \operatorname{dom} f$ and $y \in f(a)$, then there exists a set $b$ such that $b$ is finite and $b \subseteq a$ and $y \in f(b)$.

Proof: Reconsider $C=\operatorname{dom} f$ as a closed under directed unions, subsetclosed set. Reconsider $A=\{b$, where $b$ is a subset of $a: b$ is finite $\}$ as a set. $A$ is $\cup$-directed by [3, (76)], [4, (7)]. $\cup A=a$ by [3, (31)]. $A \subseteq C$. Consider $B$ being a set such that $y \in B$ and $B \in f^{\circ} A$. Consider $b$ being an object such that $b \in \operatorname{dom} f$ and $b \in A$ and $B=f(b)$. 
(2) Suppose dom $f$ is subset-closed and $f$ is preserving arbitrary unions and $\operatorname{dom} f$ is closed under directed unions. Then if $a \in \operatorname{dom} f$ and $y \in f(a)$, then there exists a set $x$ such that $x \in a$ and $y \in f(\{x\})$.

Proof: Consider $b$ being a set such that $b$ is finite and $b \subseteq a$ and $y \in$ $f(b)$. Reconsider $A=$ the set of all $\{x\}$ where $x$ is an element of $b$ as a set. $A \subseteq \operatorname{dom} f . b \subseteq \cup A$ by [3, (74), (31)]. Consider $Y$ being a set such that $y \in Y$ and $Y \in f^{\circ} A$. Consider $X$ being an object such that $X \in \operatorname{dom} f$ and $X \in A$ and $Y=f(X)$. Consider $x$ being an element of $b$ such that $X=\{x\}$.

\section{On the Union And the Intersection of Two Relational STRUCTURES}

Let $R_{1}, R_{2}$ be relational structures. The functor $\operatorname{Union}\left(R_{1}, R_{2}\right)$ yielding a strict relational structure is defined by

(Def. 1) the carrier of $i t=\left(\right.$ the carrier of $\left.R_{1}\right) \cup\left(\right.$ the carrier of $R_{2}$ ) and the internal relation of $i t=\left(\right.$ the internal relation of $\left.R_{1}\right) \cup\left(\right.$ the internal relation of $\left.R_{2}\right)$.

One can check that the functor is commutative. The functor $\operatorname{Meet}\left(R_{1}, R_{2}\right)$ yielding a strict relational structure is defined by

(Def. 2) the carrier of $i t=\left(\right.$ the carrier of $\left.R_{1}\right) \cap\left(\right.$ the carrier of $R_{2}$ ) and the internal relation of $i t=\left(\right.$ the internal relation of $\left.R_{1}\right) \cap\left(\right.$ the internal relation of $\left.R_{2}\right)$. Note that the functor is commutative.

Let $R_{1}$ be a relational structure and $R_{2}$ be a non empty relational structure. Let us observe that $\operatorname{Union}\left(R_{1}, R_{2}\right)$ is non empty.

\section{Ordinary Properties of Maps}

Let $A$ be a set. Let us note that there exists a function from $2^{A}$ into $2^{A}$ which preserves $\cap$ and $\cup$.

Let $f$ be a function from $2^{A}$ into $2^{A}$ preserves $\cap$. Observe that Flip $f$ preserves $\cup$.

Let $f$ be a function from $2^{A}$ into $2^{A}$ preserves $\cup$. Note that Flip $f$ preserves ก.

Now we state the proposition:

(3) Let us consider a non empty set $A$, and functions $f, g$ from $2^{A}$ into $2^{A}$. Suppose $f \subseteq g$. Then Flip $g \subseteq$ Flip $f$.

Proof: Set $f_{1}=$ Flip $f$. Set $\mathfrak{g}=$ Flip $g$. For every set $x$ such that $x \in \operatorname{dom} \mathfrak{g}$ holds $\mathfrak{g}(x) \subseteq f_{1}(x)$ by [15, (12)]. 
One can verify that there exists a relational structure which is non empty, mediate, and transitive.

Let $R$ be a total, mediate relational structure. One can verify that the internal relation of $R$ is mediate.

Let us consider relational structures $L_{1}, L_{2}$. Now we state the propositions:

(4) Suppose the relational structure of $L_{1}=$ the relational structure of $L_{2}$ and $L_{1}$ is mediate. Then $L_{2}$ is mediate.

(5) Suppose the relational structure of $L_{1}=$ the relational structure of $L_{2}$ and $L_{1}$ is serial. Then $L_{2}$ is serial.

Now we state the propositions:

(6) Let us consider a non empty set $A$, and functions $L, U$ from $2^{A}$ into $2^{A}$. Suppose $U=$ Flip $L$ and for every subset $X$ of $A, L(X) \subseteq L(L(X))$. Let us consider a subset $X$ of $A$. Then $U(U(X)) \subseteq U(X)$.

(7) Let us consider a non empty relational structure $R$, and elements $a, b$ of $R$. Suppose $\langle a, b\rangle \in$ the internal relation of $R$. Then $a \in \operatorname{UAp}(\{b\})$.

Let us consider a non empty relational structure $R$ and subsets $A, B$ of $R$. Now we state the propositions:

(8) $(\operatorname{UAp}(R))(A \cup B)=(\operatorname{UAp}(R))(A) \cup(\operatorname{UAp}(R))(B)$.

(9) $(\operatorname{LAp}(R))(A \cap B)=(\operatorname{LAp}(R))(A) \cap(\operatorname{LAp}(R))(B)$.

(10) Let us consider a non empty relational structure $R$. Then $(\operatorname{UAp}(R))(\emptyset)=$ $\emptyset$.

Let us consider non empty relational structures $R_{1}, R_{2}$, a subset $X$ of $R_{1}$, and a subset $Y$ of $R_{2}$.

Let us assume that the relational structure of $R_{1}=$ the relational structure of $R_{2}$ and $X=Y$. Now we state the propositions:

(11) $\operatorname{UAp}(X)=\operatorname{UAp}(Y)$.

(12) $\operatorname{LAp}(X)=\operatorname{LAp}(Y)$.

\section{On the Relational Structure Generated by Rough Approximation}

Let $R$ be a non empty relational structure and $H$ be a function from $2^{(\text {the carrier of } R)}$ into $2^{\text {(the carrier of } R)}$. The functor GeneratedRelation $(R, H)$ yielding a binary relation on the carrier of $R$ is defined by

(Def. 3) for every elements $x, y$ of $R,\langle x, y\rangle \in$ it iff $x \in H(\{y\})$.

The functor GeneratedRelStr $H$ yielding a relational structure is defined by the term 
(Def. 4) 〈the carrier of $R$, GeneratedRelation $(R, H)\rangle$.

Let us note that GeneratedRelStr $H$ is non empty.

Now we state the proposition:

(13) Let us consider a finite, non empty relational structure $R$, and a function $H$ from $2^{\alpha}$ into $2^{\alpha}$. Suppose $H(\emptyset)=\emptyset$ and $H$ preserves $\cup$. Then $\mathrm{UAp}($ GeneratedRelStr $H)=H$, where $\alpha$ is the carrier of $R$.

Proof: For every subset $A$ of $\operatorname{dom} H$ such that $\bigcup A \in \operatorname{dom} H$ holds $H(\bigcup A)=\bigcup\left(H^{\circ} A\right)$ by [3, (2)], [14, (14)], [3, (25)], [1, (59)]. Set $H_{1}=$ $\mathrm{UAp}($ GeneratedRelStr $H)$. For every subset $X$ of $R, H_{1}(X)=H(X)$ by [8, (7)], [13, (9)], [3, (31)], (2).

\section{Construction Revisited: Yao's [17] Theorem 3}

Now we state the proposition:

(14) Let us consider a finite, non empty set $A$, and functions $L, H$ from $2^{A}$ into $2^{A}$. Suppose $L=$ Flip $H$. Then $H(\emptyset)=\emptyset$ and for every subsets $X$, $Y$ of $A, H(X \cup Y)=H(X) \cup H(Y)$ if and only if there exists a non empty, finite relational structure $R$ such that the carrier of $R=A$ and $\operatorname{LAp}(R)=L$ and $\operatorname{UAp}(R)=H$ and for every elements $x, y$ of $R,\langle x$, $y\rangle \in$ the internal relation of $R$ iff $x \in H(\{y\})$.

Proof: If $H(\emptyset)=\emptyset$ and for every subsets $X, Y$ of $A, H(X \cup Y)=$ $H(X) \cup H(Y)$, then there exists a non empty, finite relational structure $R$ such that the carrier of $R=A$ and $\operatorname{LAp}(R)=L$ and $\operatorname{UAp}(R)=H$ and for every elements $x, y$ of $R,\langle x, y\rangle \in$ the internal relation of $R$ iff $x \in H(\{y\})$ by [3. (31)], [2, (5)], [3, (50), (48), (116)].

\section{Transitive Binary Relations}

Let us consider a non empty, transitive relational structure $R$ and a subset $X$ of $R$. Now we state the propositions:

(15) $\operatorname{LAp}(X) \subseteq \operatorname{LAp}(\operatorname{LAp}(X))$.

Proof: Consider $y$ being an element of $R$ such that $y=x$ and $[y]_{\alpha} \subseteq$ $X$, where $\alpha$ is the internal relation of $R$. $[y]_{\alpha} \subseteq \operatorname{LAp}(X)$, where $\alpha$ is the internal relation of $R$ by [16, (169)].

(16) $\operatorname{UAp}(\operatorname{UAp}(X)) \subseteq \operatorname{UAp}(X)$.

(17) Let us consider a finite, non empty set $A$, and a function $L$ from $2^{A}$ into $2^{A}$. Suppose $L(A)=A$ and for every subset $X$ of $A, L(X) \subseteq L(L(X))$ and for every subsets $X, Y$ of $A, L(X \cap Y)=L(X) \cap L(Y)$. Then there exists a non empty, finite, transitive relational structure $R$ such that 
(i) the carrier of $R=A$, and

(ii) $L=\operatorname{LAp}(R)$.

Proof: Set $H=$ Flip $L$. Consider $R$ being a non empty, finite relational structure such that the carrier of $R=A$ and $\operatorname{LAp}(R)=L$ and $\operatorname{UAp}(R)=$ $H$ and for every elements $x, y$ of $R,\langle x, y\rangle \in$ the internal relation of $R$ iff $x \in H(\{y\})$. For every objects $x, y, z$ such that $x, y, z \in$ the carrier of $R$ and $\langle x, y\rangle,\langle y, z\rangle \in$ the internal relation of $R$ holds $\langle x, z\rangle \in$ the internal relation of $R$ by [3, (31)], [2, (5)], (6).

(18) Let us consider a non empty, finite set $A$, and a function $U$ from $2^{A}$ into $2^{A}$. Suppose $U(\emptyset)=\emptyset$ and for every subset $X$ of $A, U(U(X)) \subseteq U(X)$ and for every subsets $X, Y$ of $A, U(X \cup Y)=U(X) \cup U(Y)$. Then there exists a non empty, finite, transitive relational structure $R$ such that

(i) the carrier of $R=A$, and

(ii) $U=\operatorname{UAp}(R)$.

The theorem is a consequence of (17).

\section{Mediate and Transitive Binary Relations}

Let us consider a non empty, mediate, transitive relational structure $R$ and a subset $X$ of $R$. Now we state the propositions:

(19) $\operatorname{LAp}(X)=\operatorname{LAp}(\operatorname{LAp}(X))$. The theorem is a consequence of (15).

(20) $\operatorname{UAp}(X)=\operatorname{UAp}(\operatorname{UAp}(X))$. The theorem is a consequence of (16).

(21) Let us consider a non empty, finite set $A$, and a function $L$ from $2^{A}$ into $2^{A}$. Suppose $L(A)=A$ and for every subset $X$ of $A, L(X)=L(L(X))$ and for every subsets $X, Y$ of $A, L(X \cap Y)=L(X) \cap L(Y)$. Then there exists a non empty, mediate, finite, transitive relational structure $R$ such that

(i) the carrier of $R=A$, and

(ii) $L=\operatorname{LAp}(R)$.

The theorem is a consequence of (17), (13), and (4).

(22) Let us consider a non empty, finite set $A$, and a function $U$ from $2^{A}$ into $2^{A}$. Suppose $U(\emptyset)=\emptyset$ and for every subset $X$ of $A, U(U(X))=U(X)$ and for every subsets $X, Y$ of $A, U(X \cup Y)=U(X) \cup U(Y)$. Then there exists a non empty, mediate, finite, transitive relational structure $R$ such that

(i) the carrier of $R=A$, and

(ii) $U=\operatorname{UAp}(R)$. 
Proof: Consider $R$ being a non empty, finite, transitive relational structure such that the carrier of $R=A$ and $U=\operatorname{UAp}(R)$. For every objects $x$, $y$ such that $x, y \in$ the carrier of $R$ holds if $\langle x, y\rangle \in$ the internal relation of $R$, then there exists an object $z$ such that $z \in$ the carrier of $R$ and $\langle x$, $z\rangle,\langle z, y\rangle \in$ the internal relation of $R$ by [3, (31)], [16, (169)], [8, (5)].

\section{Alliance Binary Relations}

Let $X$ be a set and $R$ be a binary relation on $X$. We say that $R$ is a positive alliance in $X$ if and only if

(Def. 5) for every objects $x, y$ such that $x, y \in X$ and $\langle x, y\rangle \notin R$ there exists an object $z$ such that $z \in X$ and $\langle x, z\rangle \in R$ and $\langle z, y\rangle \notin R$.

We say that $R$ is a negative alliance in $X$ if and only if

(Def. 6) for every objects $x, y$ such that $x, y \in X$ holds if there exists an object $z$ such that $z \in X$ and $\langle x, z\rangle \in R$ and $\langle z, y\rangle \notin R$, then $\langle x, y\rangle \notin R$.

We say that $R$ is an alliance in $X$ if and only if

(Def. 7) $R$ is a negative alliance in $X$ and $R$ is a positive alliance in $X$.

Let $R$ be a non empty relational structure. We say that $R$ is positive alliance if and only if

(Def. 8) the internal relation of $R$ is a positive alliance in the carrier of $R$.

We say that $R$ is negative alliance if and only if

(Def. 9) the internal relation of $R$ is a negative alliance in the carrier of $R$.

We say that $R$ is alliance if and only if

(Def. 10) the internal relation of $R$ is an alliance in the carrier of $R$.

Let us observe that every non empty relational structure which is reflexive is also positive alliance and every non empty relational structure which is discrete is also negative alliance.

There exists a non empty relational structure which is positive alliance and negative alliance and every non empty relational structure which is alliance is also positive alliance and negative alliance and every non empty relational structure which is positive alliance and negative alliance is also alliance.

Every non empty relational structure which is positive alliance is also serial and every non empty relational structure which is transitive and serial is also positive alliance.

Let us consider non empty relational structures $L_{1}, L_{2}$. Now we state the propositions:

(23) Suppose the relational structure of $L_{1}=$ the relational structure of $L_{2}$ and $L_{1}$ is negative alliance. Then $L_{2}$ is negative alliance. 
(24) Suppose the relational structure of $L_{1}=$ the relational structure of $L_{2}$ and $L_{1}$ is positive alliance. Then $L_{2}$ is positive alliance.

(25) Suppose the relational structure of $L_{1}=$ the relational structure of $L_{2}$ and $L_{1}$ is alliance. Then $L_{2}$ is alliance.

\section{Preparation for Translation of Proposition 10 (7H')}

Let $R$ be a non empty relational structure. We say that $R$ is satisfying $\left(7 \mathrm{H}^{\prime}\right)$ if and only if

(Def. 11) for every subset $X$ of $R,(\operatorname{UAp}(X))^{\mathrm{c}} \subseteq \operatorname{UAp}\left((\operatorname{UAp}(X))^{\mathrm{c}}\right)$.

We say that $R$ is satisfying (7L') if and only if

(Def. 12) for every subset $X$ of $R, \operatorname{LAp}\left((\operatorname{LAp}(X))^{\mathrm{c}}\right) \subseteq(\operatorname{LAp}(X))^{\mathrm{c}}$.

Let us consider a finite, non empty relational structure $R$. Now we state the propositions:

(26) If $R$ is satisfying (7L'), then $R$ is satisfying (7H').

Proof: For every subset $X$ of $R,(\operatorname{UAp}(X))^{\mathrm{c}} \subseteq \operatorname{UAp}\left((\operatorname{UAp}(X))^{\mathrm{c}}\right)$ by 8 , (8)], [15, (12)], [8, (9)].

(27) If $R$ is satisfying (7H'), then $R$ is serial.

Proof: Set $U=\operatorname{UAp}(R)$. For every subsets $X, Y$ of $R, U(X \cup Y)=$ $U(X) \cup U(Y)$ by [8, (13)]. Consider $S$ being a non empty, finite, serial relational structure such that the carrier of $S=$ the carrier of $R$ and $U=$ $\mathrm{UAp}(S)$.

(28) If $R$ is satisfying (7L'), then $R$ is serial.

Let us observe that every finite, non empty relational structure which is satisfying $\left(7 \mathrm{H}^{\prime}\right)$ is also serial.

Now we state the proposition:

(29) Let us consider a non empty relational structure $R$. Suppose for every subset $X$ of $R, \operatorname{UAp}\left((\operatorname{UAp}(X))^{\mathrm{c}}\right) \subseteq(\operatorname{UAp}(X))^{\mathrm{c}}$. Let us consider a subset $X$ of $R$. Then $(\operatorname{LAp}(X))^{\mathrm{c}} \subseteq \operatorname{LAp}\left((\operatorname{LAp}(X))^{\mathrm{c}}\right)$.

Let us consider a non empty set $A$, functions $L, U$ from $2^{A}$ into $2^{A}$, and a subset $X$ of $A$. Now we state the propositions:

(30) Suppose $U=$ Flip $L$ and for every subset $X$ of $A, L(X)^{\mathrm{c}} \subseteq L\left(L(X)^{\mathrm{c}}\right)$. Then $U\left(U(X)^{\mathrm{c}}\right) \subseteq U(X)^{\mathrm{c}}$.

(31) Suppose $U=$ Flip $L$ and for every subset $X$ of $A, U\left(U(X)^{\mathrm{c}}\right) \subseteq U(X)^{\mathrm{c}}$. Then $L(X)^{\mathrm{c}} \subseteq L\left(L(X)^{\mathrm{c}}\right)$.

(32) Suppose $U=$ Flip $L$ and for every subset $X$ of $A, L\left(L(X)^{\mathrm{c}}\right) \subseteq L(X)^{\mathrm{c}}$. Then $U(X)^{\mathrm{c}} \subseteq U\left(U(X)^{\mathrm{c}}\right)$. 


\section{Translation Continued}

Now we state the propositions:

(33) Let us consider a finite, positive alliance, non empty relational structure $R$, and an element $x$ of $R$. Then $(\operatorname{UAp}(R))(\{x\})^{\mathrm{c}} \subseteq(\operatorname{UAp}(R))$ $\left((\mathrm{UAp}(R))(\{x\})^{\mathrm{c}}\right)$. The theorem is a consequence of (10), (8), and (13).

(34) Let us consider a non empty, finite set $A$, and a function $U$ from $2^{A}$ into $2^{A}$. Suppose $U(\emptyset)=\emptyset$ and for every subset $X$ of $A, U(X)^{\mathrm{c}} \subseteq U\left(U(X)^{\mathrm{c}}\right)$ and for every subsets $X, Y$ of $A, U(X \cup Y)=U(X) \cup U(Y)$. Then there exists a positive alliance, finite, non empty relational structure $R$ such that

(i) the carrier of $R=A$, and

(ii) $U=\operatorname{UAp}(R)$.

Proof: Consider $R$ being a non empty, finite relational structure such that the carrier of $R=A$ and $\operatorname{LAp}(R)=$ Flip $U$ and $\operatorname{UAp}(R)=U$ and for every elements $x, y$ of $R,\langle x, y\rangle \in$ the internal relation of $R$ iff $x \in U(\{y\})$. Set $X=$ the carrier of $R$. Set $I=$ the internal relation of $R$. For every objects $x, y$ such that $x, y \in X$ and $\langle x, y\rangle \notin I$ there exists an object $z$ such that $z \in X$ and $\langle x, z\rangle \in I$ and $\langle z, y\rangle \notin I$ by [8, (7)], [16, (169)].

(35) Let us consider a non empty, finite set $A$, and a function $L$ from $2^{A}$ into $2^{A}$. Suppose $L(A)=A$ and for every subset $X$ of $A, L\left(L(X)^{\mathrm{c}}\right) \subseteq L(X)^{\mathrm{c}}$ and for every subsets $X, Y$ of $A, L(X \cap Y)=L(X) \cap L(Y)$. Then there exists a positive alliance, finite, non empty relational structure $R$ such that

(i) the carrier of $R=A$, and

(ii) $L=\operatorname{LAp}(R)$.

The theorem is a consequence of (32) and (34).

(36) Let us consider a finite, negative alliance, non empty relational structure $R$, and an element $x$ of $R$. Then $(\operatorname{UAp}(R))\left((\operatorname{UAp}(R))(\{x\})^{\mathrm{c}}\right) \subseteq$ $(\operatorname{UAp}(R))(\{x\})^{\mathrm{c}}$. The theorem is a consequence of $(10),(8)$, and (13).

Let us consider a finite, negative alliance, non empty relational structure $R$ and a subset $X$ of $R$. Now we state the propositions:

(37) $\operatorname{UAp}\left((\operatorname{UAp}(X))^{\mathrm{c}}\right) \subseteq(\operatorname{UAp}(X))^{\mathrm{c}}$.

Proof: Define $\mathcal{P}$ [subset of $R] \equiv \operatorname{UAp}\left(\left(\operatorname{UAp}\left(\$_{1}\right)\right)^{\mathrm{c}}\right) \subseteq\left(\operatorname{UAp}\left(\$_{1}\right)\right)^{\mathrm{c}}$. For every subset $B$ of $R$ and for every element $b$ of $R$ such that $\mathcal{P}[B]$ and $b \notin B$ holds $\mathcal{P}[B \cup\{b\}]$ by $[\underline{8}$, (13)], (36). For every subset $B$ of $R, \mathcal{P}[B]$ from FinSubIndA1. 
(38) $(\operatorname{LAp}(X))^{\mathrm{c}} \subseteq \operatorname{LAp}\left((\operatorname{LAp}(X))^{\mathrm{c}}\right)$. The theorem is a consequence of (37) and $(29)$.

(39) Let us consider a non empty, finite set $A$, and a function $U$ from $2^{A}$ into $2^{A}$. Suppose $U(\emptyset)=\emptyset$ and for every subset $X$ of $A, U\left(U(X)^{\mathrm{c}}\right) \subseteq U(X)^{\mathrm{c}}$ and for every subsets $X, Y$ of $A, U(X \cup Y)=U(X) \cup U(Y)$. Then there exists a negative alliance, finite, non empty relational structure $R$ such that

(i) the carrier of $R=A$, and

(ii) $U=\operatorname{UAp}(R)$.

Proof: Consider $R$ being a non empty, finite relational structure such that the carrier of $R=A$ and $\operatorname{LAp}(R)=$ Flip $U$ and $\operatorname{UAp}(R)=U$ and for every elements $x, y$ of $R,\langle x, y\rangle \in$ the internal relation of $R$ iff $x \in U(\{y\})$. Set $X=$ the carrier of $R$. Set $I=$ the internal relation of $R$. For every objects $x, y$ such that $x, y \in X$ holds if there exists an object $z$ such that $z \in X$ and $\langle x, z\rangle \in I$ and $\langle z, y\rangle \notin I$, then $\langle x, y\rangle \notin I$ by [16, (169)].

(40) Let us consider a non empty, finite set $A$, and a function $L$ from $2^{A}$ into $2^{A}$. Suppose $L(A)=A$ and for every subset $X$ of $A, L(X)^{\mathrm{c}} \subseteq L\left(L(X)^{\mathrm{c}}\right)$ and for every subsets $X, Y$ of $A, L(X \cap Y)=L(X) \cap L(Y)$. Then there exists a negative alliance, finite, non empty relational structure $R$ such that

(i) the carrier of $R=A$, and

(ii) $L=\operatorname{LAp}(R)$.

The theorem is a consequence of (30) and (39).

(41) Let us consider a non empty, finite set $A$, and a function $U$ from $2^{A}$ into $2^{A}$. Suppose $U(\emptyset)=\emptyset$ and for every subset $X$ of $A, U\left(U(X)^{\mathrm{c}}\right)=U(X)^{\mathrm{c}}$ and for every subsets $X, Y$ of $A, U(X \cup Y)=U(X) \cup U(Y)$. Then there exists an alliance, finite, non empty relational structure $R$ such that

(i) the carrier of $R=A$, and

(ii) $U=\operatorname{UAp}(R)$.

The theorem is a consequence of (39), (34), (24), and (23).

(42) Let us consider a non empty, finite set $A$, and a function $L$ from $2^{A}$ into $2^{A}$. Suppose $L(A)=A$ and for every subset $X$ of $A, L(X)^{\mathrm{c}}=L\left(L(X)^{\mathrm{c}}\right)$ and for every subsets $X, Y$ of $A, L(X \cap Y)=L(X) \cap L(Y)$. Then there exists an alliance, finite, non empty relational structure $R$ such that

(i) the carrier of $R=A$, and

(ii) $L=\operatorname{LAp}(R)$. 
Proof: Set $U=$ Flip $L$. For every subset $X$ of $A, U\left(U(X)^{\mathrm{c}}\right)=U(X)^{\mathrm{c}}$ by (30), [8, (23)], (31). Consider $R$ being an alliance, finite, non empty relational structure such that the carrier of $R=A$ and $U=\operatorname{UAp}(R)$.

\section{On the Uniqueness of Binary Relations to Generate Rough} SETS

Let us consider non empty relational structures $R_{1}, R_{2}, R$, a subset $X$ of $R$, a subset $X_{1}$ of $R_{1}$, and a subset $X_{2}$ of $R_{2}$.

Let us assume that $R=\operatorname{Union}\left(R_{1}, R_{2}\right)$ and $X=X_{1}$ and $X=X_{2}$ and the carrier of $R_{1}=$ the carrier of $R_{2}$. Now we state the propositions:

$\operatorname{UAp}(X)=\operatorname{UAp}\left(X_{1}\right) \cup \operatorname{UAp}\left(X_{2}\right)$.

Proof: $\operatorname{UAp}(X) \subseteq \operatorname{UAp}\left(X_{1}\right) \cup \operatorname{UAp}\left(X_{2}\right)$ by [16, (169)]. $\operatorname{UAp}\left(X_{1}\right) \cup$ $\operatorname{UAp}\left(X_{2}\right) \subseteq \operatorname{UAp}(X)$ by [16, (169)].

(44) $\operatorname{LAp}(X)=\operatorname{LAp}\left(X_{1}\right) \cap \operatorname{LAp}\left(X_{2}\right)$.

Proof: $\operatorname{LAp}(X) \subseteq \operatorname{LAp}\left(X_{1}\right) \cap \operatorname{LAp}\left(X_{2}\right)$ by [16, (169)]. $\operatorname{LAp}\left(X_{1}\right) \cap \operatorname{LAp}\left(X_{2}\right)$ $\subseteq \operatorname{LAp}(X)$ by [16, (169)].

Let us consider non empty relational structures $R_{1}, R_{2}$.

Let us assume that the carrier of $R_{1}=$ the carrier of $R_{2}$ and the internal relation of $R_{1} \subseteq$ the internal relation of $R_{2}$. Now we state the propositions:

(45) $\operatorname{UAp}\left(R_{1}\right) \subseteq \operatorname{UAp}\left(R_{2}\right)$.

Proof: For every set $x$ such that $x \in \operatorname{dom} \operatorname{UAp}\left(R_{1}\right)$ holds $\left(\operatorname{UAp}\left(R_{1}\right)\right)(x) \subseteq$ $\left(\operatorname{UAp}\left(R_{2}\right)\right)(x)$ by [16, (124)].

(46) $\operatorname{LAp}\left(R_{2}\right) \subseteq \operatorname{LAp}\left(R_{1}\right)$.

Proof: For every set $x$ such that $x \in \operatorname{dom} \operatorname{LAp}\left(R_{2}\right)$ holds $\left(\operatorname{LAp}\left(R_{2}\right)\right)(x) \subseteq$ $\left(\operatorname{LAp}\left(R_{1}\right)\right)(x)$ by [16, $\left.(124)\right]$.

Let us consider non empty relational structures $R_{1}, R_{2}, R$, a subset $X$ of $R$, a subset $X_{1}$ of $R_{1}$, and a subset $X_{2}$ of $R_{2}$.

Let us assume that $R=\operatorname{Meet}\left(R_{1}, R_{2}\right)$ and $X=X_{1}$ and $X=X_{2}$ and the carrier of $R_{1}=$ the carrier of $R_{2}$. Now we state the propositions:

(47) $\operatorname{UAp}(X) \subseteq \operatorname{UAp}\left(X_{1}\right) \cap \operatorname{UAp}\left(X_{2}\right)$. The theorem is a consequence of (45).

(48) $\operatorname{LAp}\left(X_{1}\right) \cup \operatorname{LAp}\left(X_{2}\right) \subseteq \operatorname{LAp}(X)$. The theorem is a consequence of (46).

Let us consider non empty relational structures $R_{1}, R_{2}$. Now we state the propositions:

(49) Suppose the carrier of $R_{1}=$ the carrier of $R_{2}$ and $\operatorname{UAp}\left(R_{1}\right) \subseteq \operatorname{UAp}\left(R_{2}\right)$. Then the internal relation of $R_{1} \subseteq$ the internal relation of $R_{2}$.

(50) Suppose the carrier of $R_{1}=$ the carrier of $R_{2}$ and $\operatorname{UAp}\left(R_{1}\right)=\operatorname{UAp}\left(R_{2}\right)$. Then the internal relation of $R_{1}=$ the internal relation of $R_{2}$. 
(51) Suppose the carrier of $R_{1}=$ the carrier of $R_{2}$. Then $\operatorname{UAp}\left(R_{1}\right)=\operatorname{UAp}\left(R_{2}\right)$ if and only if the internal relation of $R_{1}=$ the internal relation of $R_{2}$.

(52) Suppose the carrier of $R_{1}=$ the carrier of $R_{2}$ and $\operatorname{LAp}\left(R_{1}\right) \subseteq \operatorname{LAp}\left(R_{2}\right)$. Then the internal relation of $R_{2} \subseteq$ the internal relation of $R_{1}$.

(53) Suppose the carrier of $R_{1}=$ the carrier of $R_{2}$ and $\operatorname{LAp}\left(R_{1}\right)=\operatorname{LAp}\left(R_{2}\right)$. Then the internal relation of $R_{2}=$ the internal relation of $R_{1}$.

(54) Suppose the carrier of $R_{1}=$ the carrier of $R_{2}$. Then $\operatorname{LAp}\left(R_{1}\right)=\operatorname{LAp}\left(R_{2}\right)$ if and only if the internal relation of $R_{1}=$ the internal relation of $R_{2}$. The theorem is a consequence of (53) and (12).

\section{REFERENCES}

[1] Czesław Byliński. Functions and their basic properties Formalized Mathematics, 1(1): 55-65, 1990.

[2] Czesław Byliński. Functions from a set to a set Formalized Mathematics, 1(1):153-164, 1990.

[3] Czesław Byliński. Some basic properties of sets Formalized Mathematics, 1(1):47-53, 1990.

[4] Agata Darmochwał. Finite sets. Formalized Mathematics, 1(1):165-167, 1990.

[5] Adam Grabowski. On the computer-assisted reasoning about rough sets In B. DuninKẹplicz, A. Jankowski, A. Skowron, and M. Szczuka, editors, Internatıonal Workshop on Monitoring, Security, and Rescue Techniques in Multiagent Systems Location, volume 28 of Advances in Soft Computing, pages 215-226, Berlin, Heidelberg, 2005. Springer-Verlag. doi: $10.1007 / 3-540-32370-8 \_15$.

[6] Adam Grabowski. Efficient rough set theory merging. Fundamenta Informaticae, 135(4): 371-385, 2014. doi $10.3233 /$ FI-2014-1129.

[7] Adam Grabowski. Mechanizing complemented lattices within Mizar system. Journal of Automated Reasoning, 55:211-221, 2015. doi 10.1007/s10817-015-9333-5.

[8] Adam Grabowski. Relational formal characterization of rough sets. Formalized Mathematics, 21(1):55-64, 2013. doi:10.2478/forma-2013-0006.

[9] Adam Grabowski and Magdalena Jastrzębska. A note on a formal approach to rough operators. In Marcin S. Szczuka and Marzena Kryszkiewicz et al., editors, Rough Sets and Current Trends in Computing - 7th International Conference, RSCTC 2010, Warsaw, Poland, June 28-30, 2010. Proceedings, volume 6086 of Lecture Notes in Computer Science, pages 307-316. Springer, 2010. doi:10.1007/978-3-642-13529-3_33

[10] Adam Grabowski and Magdalena Jastrzębska. Rough set theory from a math-assistant perspective. In Rough Sets and Intelligent Systems Paradigms, International Conference, RSEISP 200\%, Warsaw, Poland, June 28-30, 200\%, Proceedings, pages 152-161, 2007. doi: $10.1007 / 978-3-540-73451-2 \_17$.

[11] Adam Grabowski, Artur Korniłowicz, and Adam Naumowicz. Four decades of Mizar. Journal of Automated Reasoning, 55(3):191-198, 2015. doi 10.1007/s10817-015-9345-1

[12] Zdzisław Pawlak. Rough sets. International Journal of Parallel Programming, 11:341-356, 1982. doi $10.1007 /$ BF01001956

[13] Krzysztof Retel. Properties of first and second order cutting of binary relations Formalized Mathematics, 13(3):361-365, 2005.

[14] Andrzej Trybulec and Agata Darmochwał. Boolean domains Formalized Mathematics, 1 (1):187-190, 1990.

[15] Zinaida Trybulec. Properties of subsets Formalized Mathematics, 1(1):67-71, 1990.

[16] Edmund Woronowicz. Relations and their basic properties. Formalized Mathematics, 1 (1):73-83, 1990.

[17] Y.Y. Yao. Two views of the theory of rough sets in finite universes. International Journal 
of Approximate Reasoning, 15(4):291-317, 1996. doi 10.1016/S0888-613X(96)00071-0.

[18] William Zhu. Generalized rough sets based on relations. Information Sciences, 177: 4997-5011, 2007.

Received February 15, 2016 